\title{
A digital method for separation and reconstruction of pile-up events in germanium detectors
}

\author{
M. Nakhostin ${ }^{\text {a) }}$, Zs. Podolyak, P. H. Regan, P. M. Walker \\ Department of Physics, University of Surrey, Guildford GU2 7XH, UK
}

\begin{abstract}
The problem of pulse pile-up is very often encountered in precise measurements of $\gamma$ rays using germanium detectors. The standard method of treating the pile-up events is to identify and reject them using an appropriate electronics system. Digital acquisition techniques now allow the recording of waveforms of pile-up events that can be analysed and the contributing single pulses recovered, rather than simply tolerating the losses associated with pile-up. In this paper, a method for the off-line digital processing of pile-up events from germanium detectors is demonstrated. The method is based on an appropriate fitting of the detector signals, shaped with a suitable digital pulse shaper. It is shown that the method is able to recover the pile-up events with good accuracy even when the constituent signals are in close proximity. The method is very useful for $\gamma$-ray spectroscopy in nuclear physics experiments, where the low intensity signals can be lost due to the pile-up in a high-rate environment.
\end{abstract}

PACS: 29.30.Kv, 29.85.Fj

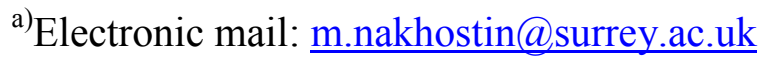




\section{INTRODUCTION}

The development of nuclear structure studies has depended to a large extent upon the $\gamma$-ray spectroscopy technique using germanium detectors. Germanium arrays ${ }^{1}$ like AGATA, GAMMASPHERE, EXOGAM have permitted detailed studies of nuclear structure and this field of research is now pushed to more extreme cases making use of the available and future radioactive beam facilities. In such experiments, germanium detectors often work at high count rates of $\mathrm{X}$ and $\gamma$-rays coming not only from the target but also from the beam interactions with surroundings. This leads to a high fraction of pile-up events, which are events in which two or more pulses partly or completely overlap in time. If not properly recognized, these signals are interpreted as single events, with an energy equal to the sum of the constituent pulses, with a resulting degradation of the pulse height spectrum. There are several methods which allow the identification and rejection of pile-up events, thus restoring to a good extent the original energy resolution. These techniques, however, can not be satisfactorily applied when pile-up signals constitute a large fraction of the detected events. Moreover, in experiments aiming at the detection of low-intensity energy lines, in order to increase the counting statistics, it is desirable to record and analyze all events including those that give rise to pile-up.

The availability of digital pulse processing systems has opened the possibility of separating and analysing the pile-up events rather than simply tolerating the losses associated with pile-up. Digital acquisition techniques have been used to record waveforms of pile-up events that can be analyzed by de-convolution ${ }^{2}$ or fitting methods $^{3}$, in order to recover the original single-pulse information. In this paper, we present a digital method for processing the pile-up events in germanium detectors. The method is based on an appropriate fitting of the detector signals, shaped with a suitable digital pulse shaper. The analysis is presented for pile-up events formed by two signals, and can be extended to multiple pile-up events, as well. It is shown that the method is capable of separating the pile-up events with good accuracy, even for the cases that the pulses are in close proximity.

\section{EXPERIMENTAL SETUP}

The experimental setup consists of a cylindrical germanium detector connected to a charge-sensitive preamplifier. The hardware used for data collection is a product of 
$\mathrm{X}$-ray Instrument Associates (XIA) ${ }^{4}$. The preamplifier signals are sampled as voltage values in XIA's DGF Pixie-4 acquisition card at 75MHz with 14-bit ADCs. Test experiments were performed using a ${ }^{137} \mathrm{Cs}$ source with $400 \mathrm{kBq}$ activity and events were recorded over timescales $14 \mu$ s in length, which leads to approximately 1000 samples per event. The digitized waveforms are saved on a disk drive and transferred to a PC for offline analysis. The offline analysis is performed using a program written in MATLAB language.

\section{PRINCIPLE OF THE METHOD}

To describe the developed algorithm, we start with a brief description of pulse processing in $\gamma$-ray spectroscopy systems. In such systems, signals from a preamplifier are shaped by a pulse shaper, to optimize the signal-to-noise ratio. Among the different methods of pulse shaping, Gaussian pulse shaping is a common choice due to its simplicity and good signal-to-noise ratio. Theoretically, a Gaussian pulse shaper is composed of a differentiation stage followed by an infinite number of integration stages. In practice, a limited number (n) of integration stages is used, called the filter order. If the differentiation and $n$ integration time constants are all the same value $\tau$, the response function of the corresponding circuit is described by ${ }^{5}$ :

$$
E_{\text {out }}(t)=\frac{E}{n !}\left(\frac{t-t_{0}}{\tau}\right)^{n} e^{\frac{-\left(t-t_{0}\right)}{\tau}}
$$

where $t_{0}$ is the signal start-time and $E$ is the signal amplitude. In practise, normally four stages of integration $(n=4)$ are used and the resulting $C R-(R C)^{4}$ filter is called a semi-Gaussian filter, as its output is only an approximation of the Gaussian shape. Because the semi-Gaussian filter is a linear filter, its output for a pile-up event consisting of two pulses can be expressed as the sum of two semi-Gaussian functions. In our method, a digital pile-up detector is employed to detect the pile-up events. When a pile-up event consisting of two separate signals is detected by the pile-up detector, the signal which is appropriately shaped by a digital semi-Gaussian pulse shaper is fitted by the sum of two semi-Gaussian functions and the amplitudes of the individual signals are determined as fitting parameters. Since a fit according to Eq.1 with several free parameters is CPU time consuming and may not always easily lead to the best resolution, to simplify the fitting procedure and improve the fitting accuracy, it is necessary to reduce the number of free parameters by identifying their 
single best value or finding a relation between them. Moreover, in order to minimize the fitting error, special care should be paid to the pulse shaping process to obtain a pure semi-Gaussian signal. The details of the pulse shaping process and fixing the parameters involved in the fitting procedure are described in the section A. The algorithm used to identify the pile-up events is described in section B.

\section{A. Pulse shape analysis}

Fig. 1(a) shows a typical signal from the germanium detector. The preamplifier output exponentially decays with the preamplifier decay time constant $T_{\mathrm{f}}$. Shaping of such a pulse with the semi-Gaussian filter usually results in an undershoot in the unipolar pulse. This deviation from the semi-Gaussian pulse shape will cause a significant error in the amplitude determination using the fitting procedure. To obtain a perfect unipolar semi-Gaussian pulse, first the preamplifier signal is corrected for the finite decay-time $T_{f}$ and converted into a step function. The correction for the finite decay-time $\mathrm{T}_{\mathrm{f}}$ is done using the following relation:

$u_{k+1}=u_{k}+\left(v_{k+1}-v_{0}\right) e^{\frac{\Delta t}{2 T_{f}}}-\left(v_{k}-v_{0}\right) e^{\frac{-\Delta t}{2 T_{f}}}$

Where $u_{k}$ and $v_{k}$ are the signal samples before and after the correction and $\Delta t$ is the signal sampling interval. The preamplifier decay-time constant $\mathrm{T}_{\mathrm{f}}$ is determined by fitting an exponential function to the preamplifier signals. Fig. 1(b) shows the signal after correction of the preamplifier decay-time. The resulting step signal is fed to the digital semi-Gaussian pulse processing algorithm. The signal after shaping with the semi-Gaussian filter together with the result of fitting with Eq.1 is shown in Fig. 1(c). The fitting was done by setting the signal amplitude $\mathrm{E}$, the start-time $\mathrm{t}_{0}$ and the filter order $\mathrm{n}$ as free parameters and the $\tau$ was set $600 \mathrm{~ns}$, corresponding to $2.4 \mu$ s total shaping time constant. The fitting procedure was applied to a significant number of events and it was observed that the signals are accurately fitted by the Eq. 1 even for low-energy events. The average value of the filter order (n) was determined as $n=3.96477$, which is very close to the theoretical value of the filter order $(n=4)$. This enables the filter order to be fixed and, therefore, only two free parameters, $E$ and $t_{0}$, remain. In the next step, the signal start-time $\mathrm{t}_{0}$ is determined by employing a digital 
pulse timing method such as constant-fraction discrimination (CFD). In fact, timing methods are able to determine the start-time of pulses from germanium detectors with an uncertainty of less than $10 \mathrm{~ns}$, which is sufficient for our fitting procedure in which the fitting range of energy signals is several $\mu$ s. The details of the determination of the signal start-time using the CFD technique are illustrated in Fig. 2. The signals are first shaped by the semi-Gaussian $\left(\mathrm{CR}-(\mathrm{RC})^{4}\right)$ filter with a small shaping time constant (here $40 \mathrm{~ns}$ ) in order to reduce the noise and signal duration. The CFD method then determines the signal start-time by analysing the signal to determine when the signal crosses a predefined threshold. The threshold is set as a fraction of the signal's amplitude. While the true value of the signal's start-time is determined by using lower CFD thresholds, a low threshold can cause timing error by triggering on the signal noise. This is particularly the case for a signal whose amplitude exceeds the noise level only by a small amount. Multiple analyses were performed to study the effect of the threshold value on the fitting accuracy. It was found that a threshold value equal to the $20 \%$ of the signal maximum value is a good choice, leading to very good fitting accuracy and minimizing the effect of noise. After determining the signal start-time, the pulse amplitude remains as the only parameter to be determined by the fitting procedure.

\section{B. Pile-up detector}

A pile-up detector is required to identify the pile-up events. A common method for pile-up inspection in $\gamma$-ray spectroscopy systems is to process the signals in a fast channel in parallel to the slow pulse processing one ${ }^{6,7}$. In the fast channel, the signals are differentiated to form narrow pulses and the pile-up detector examines the pile-up events by measuring the time intervals between these signals. If the time between successive signals is less than a predefined value, the event is recognized as a pile-up event and the corresponding signal in the slow channel is rejected. However, as will be shown later, this method fails to detect a pile-up event when two signals happen in very close proximity or when one of the signals has small amplitude. In order to more effectively detect the pile-up events, we have used a different method which analyses the shape of signals after the semi-Gaussian pulse shaper. The details of this method are shown in Fig. 3. The method calculates the width of a signal at $20 \%$ of its maximum value and compares it with an average value calculated using a considerable number of single events. If the pulse width is larger than the average 
value, it is considered as pulse pile-up. The advantage of this method is that it acts on the shaped signals and, therefore, is less sensitive to the electronic noise.

\section{SIMULATION OF THE METHOD}

In order to check the accuracy of the method for pile-up separation, a number of pile-up events were generated starting from individual signals recorded with the ${ }^{137} \mathrm{Cs}$ source. Pairs of single events were first shaped by the semi-Gaussian filter with $2.4 \mu \mathrm{s}$ shaping time constant and their amplitudes were determined. Then, the preamplifier signals were added together with a time separation varying between $3 \mu$ s and $0.25 \mu \mathrm{s}$ in steps of $0.25 \mu$ s and the resulting pile-up events were shaped by the semi-Gaussian filter. The outputs of the semi-Gaussian filter were fit with a sum of two semiGaussian functions and the resulting amplitudes were compared with the original amplitudes of the signals. In the fitting procedure, the start-times of the signals constituting the pile-up events were determined through the CFD timing method, explained in the previous section, and the filter order was fixed as 3.96477. The difference between the amplitude of two typical signals determined by the fitting procedure and their original value as a function of the time difference between overlapping signals are shown in Fig. 4. The original amplitudes of the signals are approximately 600 and $200 \mathrm{keV}$. It is seen that for the pile-up events, composed of signals with a time difference greater than $250 \mathrm{~ns}$, the method is able to accurately determine the signals' amplitudes and the associated error is less than $1 \%$. However, the error increases, when the two signals are very close to each other. This is because the reconstruction of both components in a pile-up event depends primarily on the accuracy of the signals' start-times and for the close signals, the shape of the second signal is affected by the tail of the first signal which causes an error in the determination of the start-time of the second signal. This error can be minimized by using smaller shaping time constants, but the choice of shaping time constant is limited by the effect of electronic noise and therefore a compromise should be made.

\section{APPLICATION OF THE METHOD TO PILE-UP EVENTS}

Fig. 5 shows a spectrum collected when the ${ }^{137}$ Cs source is placed very close to the detector surface. The spectrum was obtained by shaping the signals with a $2.4 \mu \mathrm{s}$ shaping time constant. The interaction rate is approximately $100 \mathrm{kHz}$. In spite of the 
high rate of $\gamma$-ray interactions with the detector, very few pile-up events are seen in the spectrum due to the pile-up rejection by the built-in pile-up rejector of the data acquisition system. The few remaining pile-up events are either from the events consisting of pulses at very close proximity or small amplitudes. Fortunately, the majority of these events are detected by our pile-up detection algorithm described in section III. Around 600 such pile-up events were identified among the sixty thousand collected events. These pile-up events were processed by the timing measurement algorithm to determine the start-time of the pulses constituting the pile-up event and then the pile-up waveforms were fit using the fitting program. Two typical examples of the pile-up events are shown in Fig. 6. The waveforms correspond to two extreme cases of pile-up where one of the overlapping signals has small amplitude (Fig. 6(a)) or the signals are very close to each other (Fig 6(b)). The spectrum obtained from the separated events is shown in Fig.7. A low-energy peak due to the recovered X-rays and a full energy peak $(662 \mathrm{keV})$ are clearly seen. It is interesting that the X-rays peak appears in the recovered spectrum despite the fact that the X-ray amplitude is below the energy threshold of the system. The spectrum has an energy resolution of $2.2 \%$ at $662 \mathrm{keV}$. This resolution is rather poor, compared to the intrinsic resolution of the detector. However, the fact that these separated events are the extreme cases of very close events or small amplitude events implies that a better performance can be expected for the actual experiments when all pile-up events are included. It should be emphasised that the spectrum is obtained by processing all the pile-up events not detected by the pile-up detector of XIA system, down to minimum distance between signals of approximately $250 \mathrm{~ns}$.

\section{CONCLUSIONS}

In conclusion, we have presented in this paper a powerful method for separating the pile-up events in germanium detectors. The simulation and test experiment results demonstrate that the method is able to recover pile-up events with good accuracy. The method is very useful for recovering low-intensity events which are lost due to pulse pile-up in a high intensity background.

\section{ACKNOWLEDGMENTS}

We acknowledge support from the UK STFC and AWE plc. 

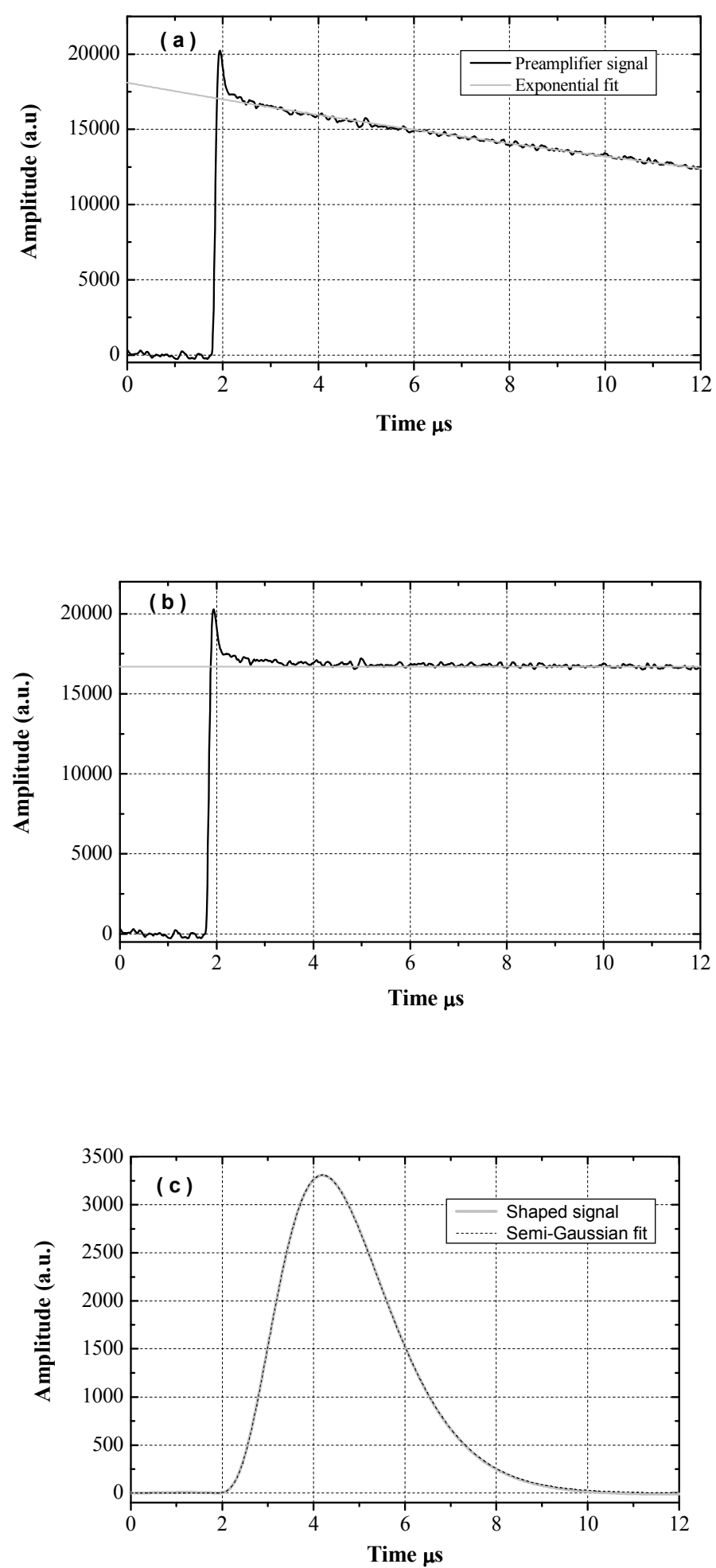

FIG.1. (a) A typical preamplifier output signal for a $\gamma$-ray interaction with $662 \mathrm{keV}$ energy. The decay time constant of the preamplifier $T_{f}$ is determined by an exponential fit to the decaying part of the signal as $44 \mu \mathrm{s}$, (b) the step pulse obtained after the correction of preamplifier decay-time constant, (c) the output of the semi- 
Gaussian filter with $2.5 \mu$ s shaping time constant (gray curve) and its fit with the semi-Gaussian function (dashed curve). The signal and the fit overlap almost completely.

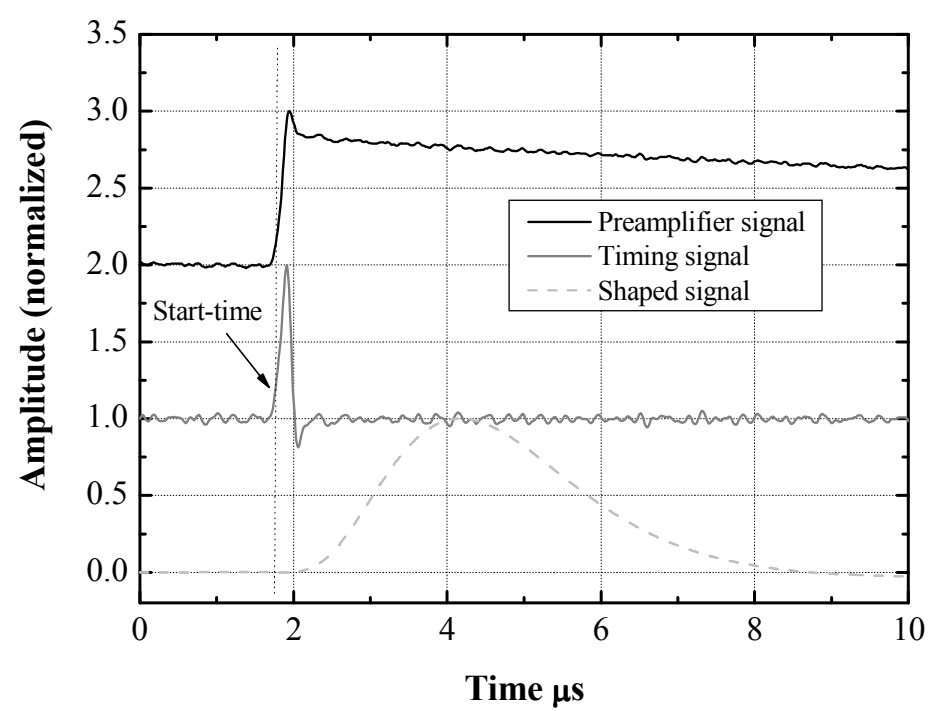

FIG. 2. Details of the determination of signal start-time $\left(\mathrm{t}_{0}\right)$. (Top) preamplifier signal. (middle) timing signal obtained by passing the signal through the semi-Gaussian shaper with a short shaping time constant (40 ns). The start-time of the signal is decided by the time that the signal reaches $20 \%$ of its amplitude. (Bottom) the relation between the signal start-time and shaper output.

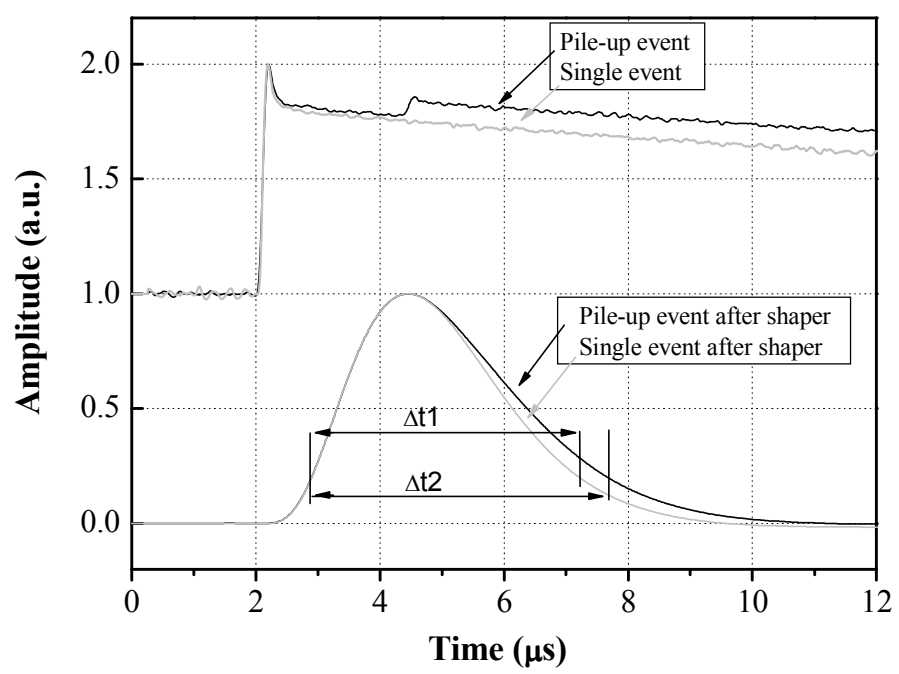

FIG. 3. The details of the pile-up detection algorithm. (Top) preamplifier signal for a pile-up event and a single event. (Bottom) the shaper outputs for the single event and the pile-up event. The shaper has $2.4 \mu$ s shaping time constant. The pile-up event has a greater width $(\Delta t 2)$ than the width of the single event $(\Delta t 1)$. This property is used for 
pile-up detection. For the signals shaped with $2.4 \mu$ s shaping time constant, the average value of the signal's width was calculated to be $4.480 \pm 0.027 \mu$ s at $20 \%$ of the signal's amplitude. Therefore, any signal with a width greater than $4.507 \mu \mathrm{s}$ is considered as pile-up.

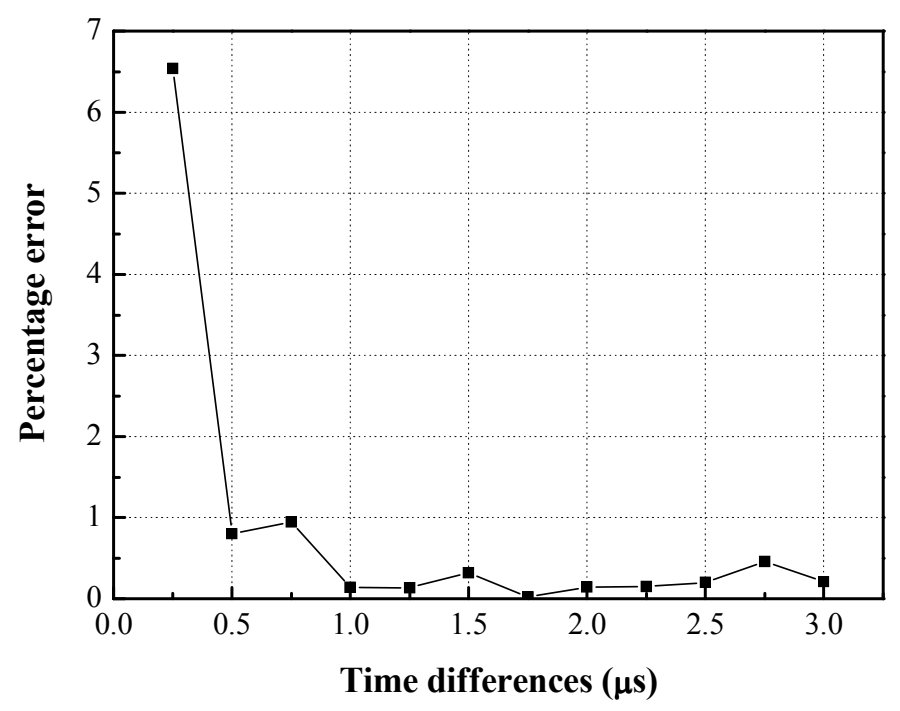

FIG. 4. Percentage error in the calculation of pulse amplitude as a function of distance between the individual signals. The amplitudes of the signals are approximately 600 and $200 \mathrm{keV}$. The same trend is observed for the second signal.

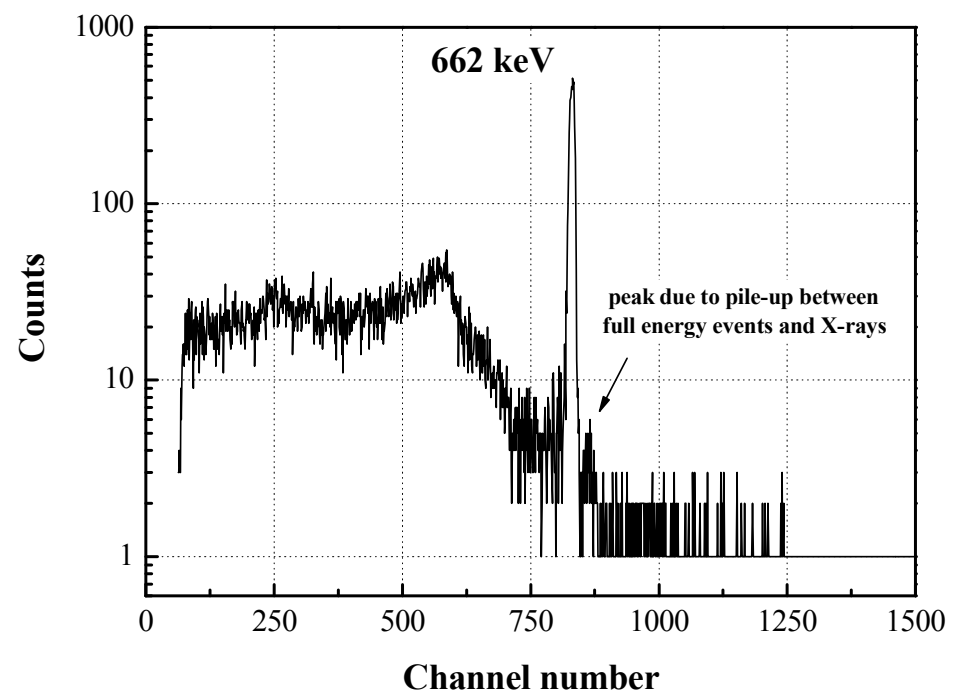

FIG. $5 .{ }^{137} \mathrm{Cs}$ energy spectrum obtained by the standard semi-Gaussian filter. The small peak next to the full energy peak is due to the pile-up between the X-rays and full energy events of the ${ }^{137} \mathrm{Cs}$ source. 


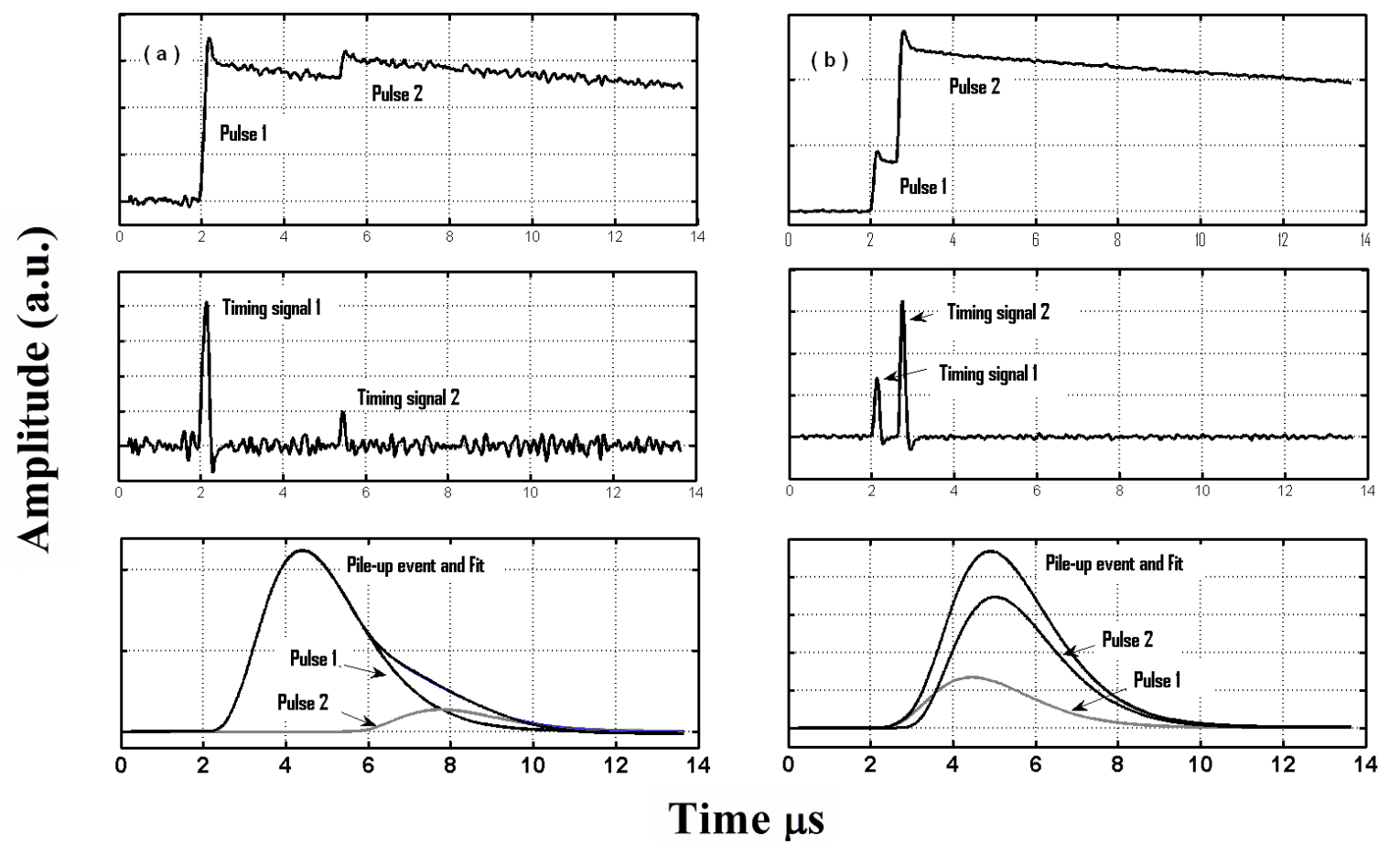

FIG. 6. Two typical examples of recovered pile-up events. Top: the pile-up events at preamplifier output. Middle: the timing signals obtained by passing the preamplifier signal through a semi-Gaussian filter with short shaping time constants (40 ns). Bottom: the separated signals together with the pile-up events and their corresponding fits.

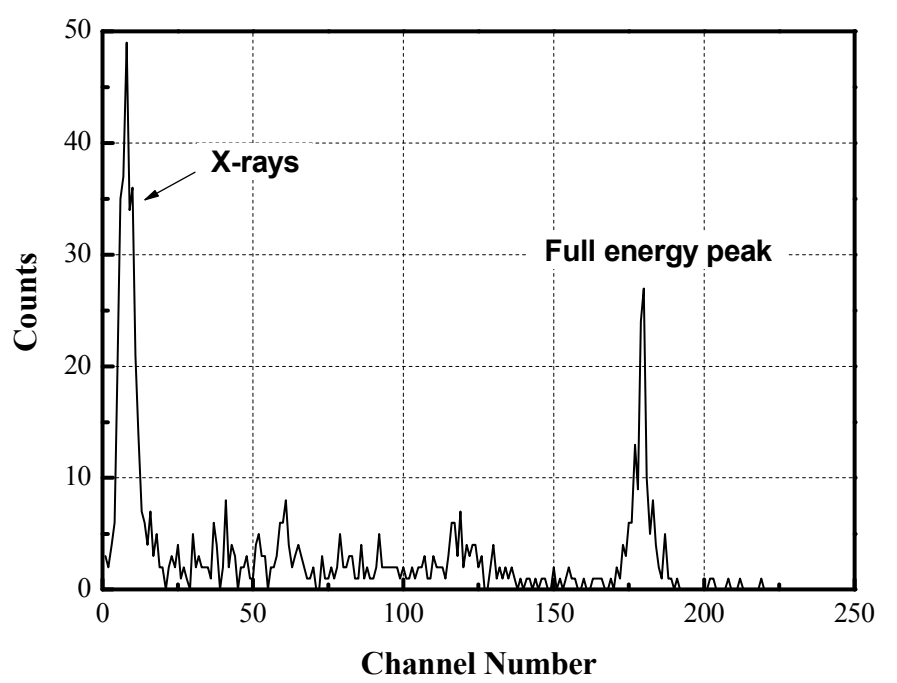

FIG. 7. Energy spectrum of ${ }^{137} \mathrm{Cs}$ obtained by separation of pile-up events. The low energy peak is due to the recovered X-rays. An energy resolution of $2.2 \%$ at $662 \mathrm{keV}$ is achieved. 
${ }^{1}$ J. Eberth, J. Simpson, Progress in Particle and Nuclear Physics, 60, 283 (2008).

${ }^{2}$ Weijun Guo, Robin P. Gardner, Charles W. Mayo, Nucl. Instrum. Methods Phys. Res. A 544, 668 (2005).

${ }^{3}$ F. Belli, B. Esposito, D. Marcco, M. Riva, Y. Kaschuck, G. Bonheure, JET EFDA contributors, Nucl. Instrum. Methods Phys. Res. A 595, 519 (2008).

${ }^{4} \mathrm{http}: / / \mathrm{www} \cdot \mathrm{xia} \cdot \mathrm{com} /$

${ }^{5}$ G. F. Knoll, Radiation Detection and Measurements ( Wiley, New York, 2000 ).

${ }^{6}$ M. Moszyński, J. Jastrzebski, B. Bengtson, Nucl. Instrum. and Methods Phys. Res. 47, 61(1967).

${ }^{7}$ M. Bolic, V. Drndarevic, Nucl. Instrum. and Methods Phys. Res. 482,761 ( 2002). 